\title{
Entrelacs
}

Cinéma et audiovisuel

$10 \mid 2013$

Le Toucher

Toucher la plaie. Tactilité de la visualité chrétienne de L'Incrédulité de Saint Thomas du Caravage à Mourir comme un homme de Joao Pedro Rodrigues

\section{Olivier Cheval}

\author{
(2) OpenEdition \\ Journals \\ Édition électronique \\ URL : http://journals.openedition.org/entrelacs/487 \\ DOI : $10.4000 /$ entrelacs.487 \\ ISSN : 2261-5482 \\ Éditeur \\ Éditions Téraèdre
}

Référence électronique

Olivier Cheval, « Toucher la plaie. Tactilité de la visualité chrétienne de L'Incrédulité de Saint Thomas du Caravage à Mourir comme un homme de Joao Pedro Rodrigues », Entrelacs [En ligne], 10 | 2013, mis en ligne le 11 septembre 2013, consulté le 10 décembre 2020. URL : http://journals.openedition.org/ entrelacs/487; DOI : https://doi.org/10.4000/entrelacs.487

Ce document a été généré automatiquement le 10 décembre 2020.

Tous droits réservés 


\title{
Toucher la plaie. Tactilité de la visualité chrétienne de L'Incrédulité de Saint Thomas du Caravage à Mourir comme un homme de Joao Pedro Rodrigues
}

\author{
Olivier Cheval
}

1 «Si je ne vois pas dans ses mains la marque des clous, si je n'enfonce pas mon doigt à la place des clous et si je n'enfonce pas ma main dans son côté, je ne croirai pas. » (Jn, 20-25). Pour croire l'incroyable, l'apôtre Thomas demande une preuve; cette preuve, Jésus la lui présente en personne, en lui offrant de constater les stigmates de sa crucifixion: "Avance ton doigt ici et regarde mes mains; avance ta main et enfonce-la dans mon côté, cesse d'être incrédule » (Jn, 20-27). Thomas obéit et reconnaît son Seigneur, qui prononce alors son ultime maxime: «Parce que tu m'as vu, tu as cru; bienheureux ceux qui, sans avoir vu, ont cru ». (Jn, 20-29).

2 La preuve que réclame Thomas pour croire en la résurrection du Christ, le visible seul ne pouvait la lui donner. Thomas a besoin de toucher: il a besoin de la matérialité d'un contact, de la profondeur d'une pénétration. Pourtant, la sentence générale qu'en tire le Christ réduit le geste tactile de Thomas à un acte de vision. Il y a là l'indice d'une confusion, d'un recouvrement du toucher par le voir dans la pensée chrétienne : le tangible n'est pas séparé du visible, il est comme sa promesse. La visualité chrétienne est une haptologie ou un continuisme, au sens derridien ${ }^{1}$ : seule est pleine, accomplie et certaine la vision qui débouche sur le contact. Voir est tendu vers le toucher, et ce jusqu'à leur indifférenciation: l'ordre christique «Avance ton doigt ici et regarde mes mains » semble être l'invitation à un regard digital, à une vision manuelle.

Cette croyance en la tactilité du visible n'est pas séparable d'une pensée de la chair. Toucher le corps du Christ, pour Thomas, c'est confirmer la présence corporelle que la 
vision induit mais ne certifie pas : c'est dissiper le soupçon d'un mirage. Ce fut d'ailleurs le grand combat théologique d'un des premiers Pères de l'Église chrétienne, Tertullien, que de lutter contre les hérésies gnostiques qui faisaient de Jésus un corps éthéré - un non-corps de nature spirituelle ou de naissance astrale ${ }^{2}$ - pour faire du christianisme la religion de l'incarnation qui oppose aux images parfaites des dieux grecs et à la Face invisible de Yahvé un Dieu de chair et de sang, né des entrailles d'une femme et mort dans la souffrance. La chair est cette profondeur matérielle et sensible qui vient doubler l'image du corps; elle est cette réalité que je peux toucher, au double sens du mot : un corps physique sur lequel je peux mettre la main, et une vie que je peux émouvoir. Toucher, ce peut être caresser, pénétrer ou blesser.

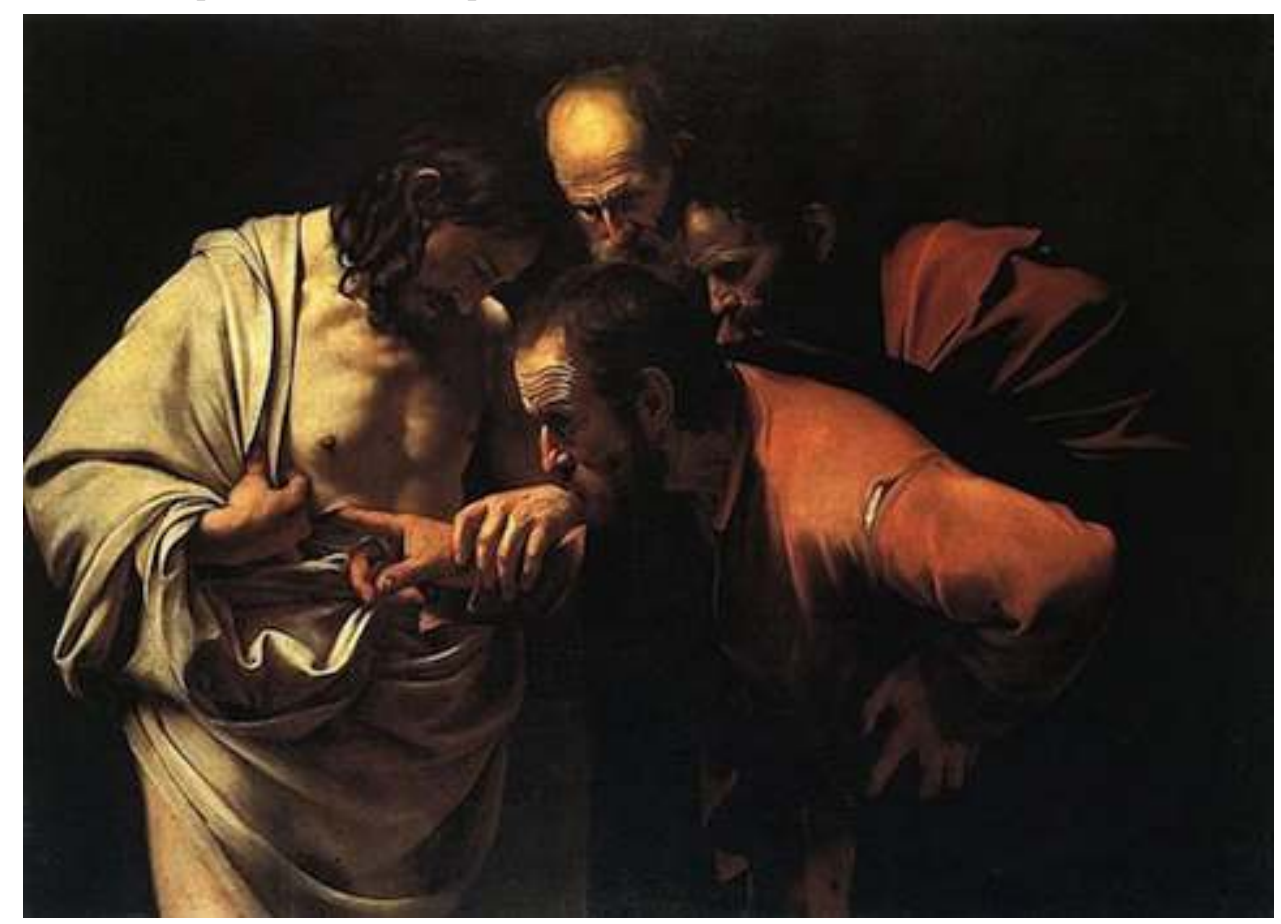

Fig. 1 : L'incrédulité de Saint Thomas, Le Caravage (vers 1602)

Dans L'incrédulité de Saint Thomas du Caravage, peinte vers 1602 (fig.1), plus que dans toute autre représentation de cet épisode de l'Évangile de Jean (par exemple la gravure de Dürer de 1509), la tactilité du voir chrétien est l'enjeu même du tableau. Par le resserrement de la scène autour de trois apôtres seulement (Saint Jean et Saint Pierre se tiennent derrière Saint Thomas pour observer son geste) regroupés en un ensemble compact, par le « choix inhabituel, et peut-être sans précédent, d'un format horizontal et d'un cadrage aux trois quarts ${ }^{3}$ ", et par la crudité d'une lumière frontale qui isole le cercle des quatre corps d'un fond uniformément obscur, le peintre concentre sa dramaturgie sur le geste de pénétration de la plaie et réduit le tableau à quatre corps qui regardent, touchent et sont touchés.

5 L'oeuvre entier du Caravage a inventé un ténébrisme qui découpe des chairs blanches, éclairées crûment, sur un fond noir qui ne laisse rien deviner de l'étendue visible d'un monde. L'adaptation de l'épisode évangélique est l'occasion pour lui de figurer, presque à la manière d'un art poétique, la pensée du visible que produit cette nouvelle manière du clair-obscur. Envisageant l'oeuvre du Caravage dans sa globalité, Louis Marin a bien noté la radicalité figurative des noirs où sont plongés ses figures : d'une part, en termes de représentation, l'espace scénique devient un « espace arcanien", «celui du coffre, du 
cercueil, de la cellule, l'espace totalement borné et délimité, celui du tombeau fermé et scellé dont on ne peut savoir - voir - ce qui s'y passe ${ }^{4} »$; d'autre part, plastiquement, «le fond noir est beaucoup plus qu'un 'fond' d'espace scénique. Le fond est à la limite la surface même du tableau. Par suite la projection du faisceau lumineux dans le plan du tableau ne laisse à la disposition des figures que l'extrême bord de la surface, la première ligne du tableau: le sol de la scène est une avant-scène et les figures sont continûment poussées vers l'avant, à peu près comme si nous avions affaire à des figures en relief sur une paroi solide, la paroi du tombeau arcanien ${ }^{5} »$.

6 Cette description générale du ténébrisme caravagesque semble être la vérité figurée par le tableau dans la situation même qu'il représente : Thomas cherche à vérifier que le Christ a bien ressuscité, qu'il est bien sorti du tombeau, et pourtant Le Caravage situe la scène dans ce non-lieu obscur qui parait être l'intérieur même d'un espace tombal. Tandis que le peintre montre le visible là où il ne devrait pas apparaître - dans le noir scellé du cercueil -, Thomas cherche à voir la réalité sans forme de l'intérieur d'une plaie et la réalité sans représentation du miracle de la résurrection. Le peintre organise donc la mise en échec de l'œil, qui cède son pouvoir à la main : main ou manière du peintre qui fait voir la nuit invisible du tombeau, main de Thomas qui prend le relais d'un œil trop lointain

7 Ce que figure le tableau, c'est l'aporie d'un voir toujours porté vers une proximité plus grande, un contact plus vrai, une pénétration sans fin. De même que le fond noir qui fonctionne comme quasi surface rapproche les quatre figures du spectateur, de même les quatre spectateurs représentés- dont le Christ lui-même, qui regarde sa propre plaie baissent la tête pour voir au plus près ; les trois apôtres ont le corps plié pour rapprocher l'œil de la côte. Thomas a d'ailleurs le corps si courbé et la tête si avancée que sa bouche arrive juste devant la main que le Christ a posé sur la sienne pour la guider vers la plaie. Le problème figuratif du tableau peut donc s'exprimer ainsi : de quel geste sommes-nous le témoin, nous qui regardons avec et en face de ces quatre acteurs-spectateurs ? Thomas touche la plaie, mais Jésus dirige la main droite de Thomas avec sa main gauche; et la pénétration violente de la plaie semblable à deux lèvres est redoublée par l'impression visuelle que Thomas baise la main qui le guide. Le doigt semble écarter ces deux lèvres, mais, en écho au baiser virtuel, et vu le geste directif de Jésus, il est possible de penser que ces deux lèvres avalent le doigt. Surtout que la lumière, qui vient de la gauche du tableau, de l'arrière du Christ, du lieu et du temps d'où il arrive - le tombeau de la résurrection - ne vient éclairer que d'autres plis : le drapé blanc de son habit, qu'il ouvre de la main droite pour découvrir la plaie, les rides des trois fronts stupéfaits des apôtres, et surtout la petite déchirure du tissu à l'épaule gauche de Thomas, de même taille que le stigmate costal, détail qui vient signer figuralement la condensation des deux motifs, la plaie et la bouche, comme ouverture et déchirure qui fait face aux huit yeux avides.

8 Il y aurait ainsi une série gestuelle qui dessinerait une chaine d'intensification du voir qui aurait comme coordonnées l'œil, la main et la bouche et comme mouvements le passage du regard au toucher et du toucher au manger : voir, se pencher pour mieux voir, se rapprocher pour voir plus près, toucher, pénétrer, ouvrir, baiser, avaler, incorporer. Autant de gestes qui trouvent dans l'Eucharistie (confirmée lors du Concile de Trente en 1563, soit huit année avant la naissance du peintre) la ritualisation de cette logique, logique dont Caravage montre la violence: voir véritablement, c'est être avalé par le visible scruté. Le corps courbé et le front plissé des apôtres marquent peut-être moins l'effort pour voir que la résistance à l'éblouissement de la vérité : dans la nuit du tombeau, la lumière venue de la résurrection met les chairs à nu et dévoile l'évidence 
opaque du miracle, évidence aussi sèche que la plaie exsangue, semblable à un trou de sens qui aspire les corps. Vérité figurée crûment dans cet espace hors du monde, dans cette avant-scène, par le geste immonde, obscène du doigt qui entre dans la plaie, l'écarte, s'y enfonce et s'y perd.

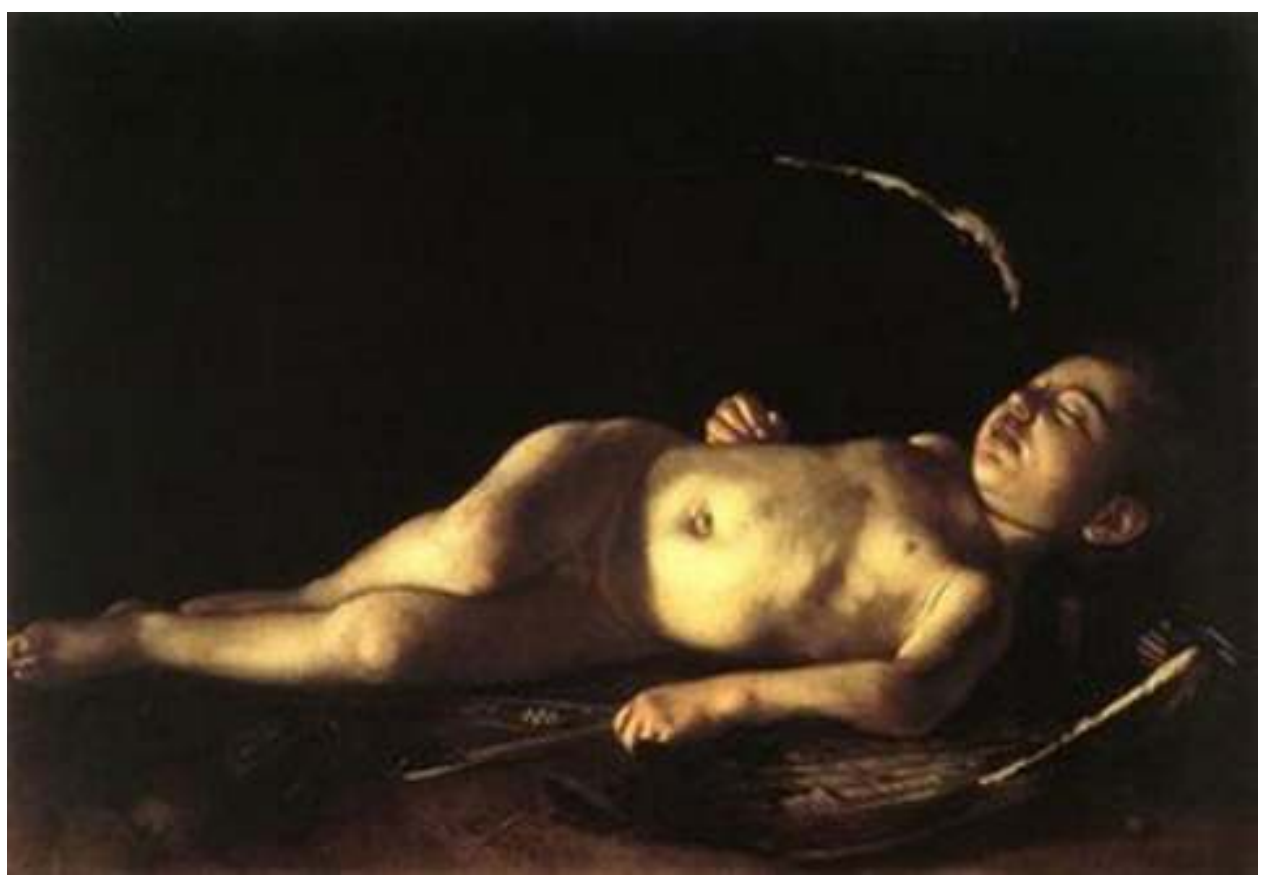

Fig. 4 : L'amour endormi, Le Caravage (1608)

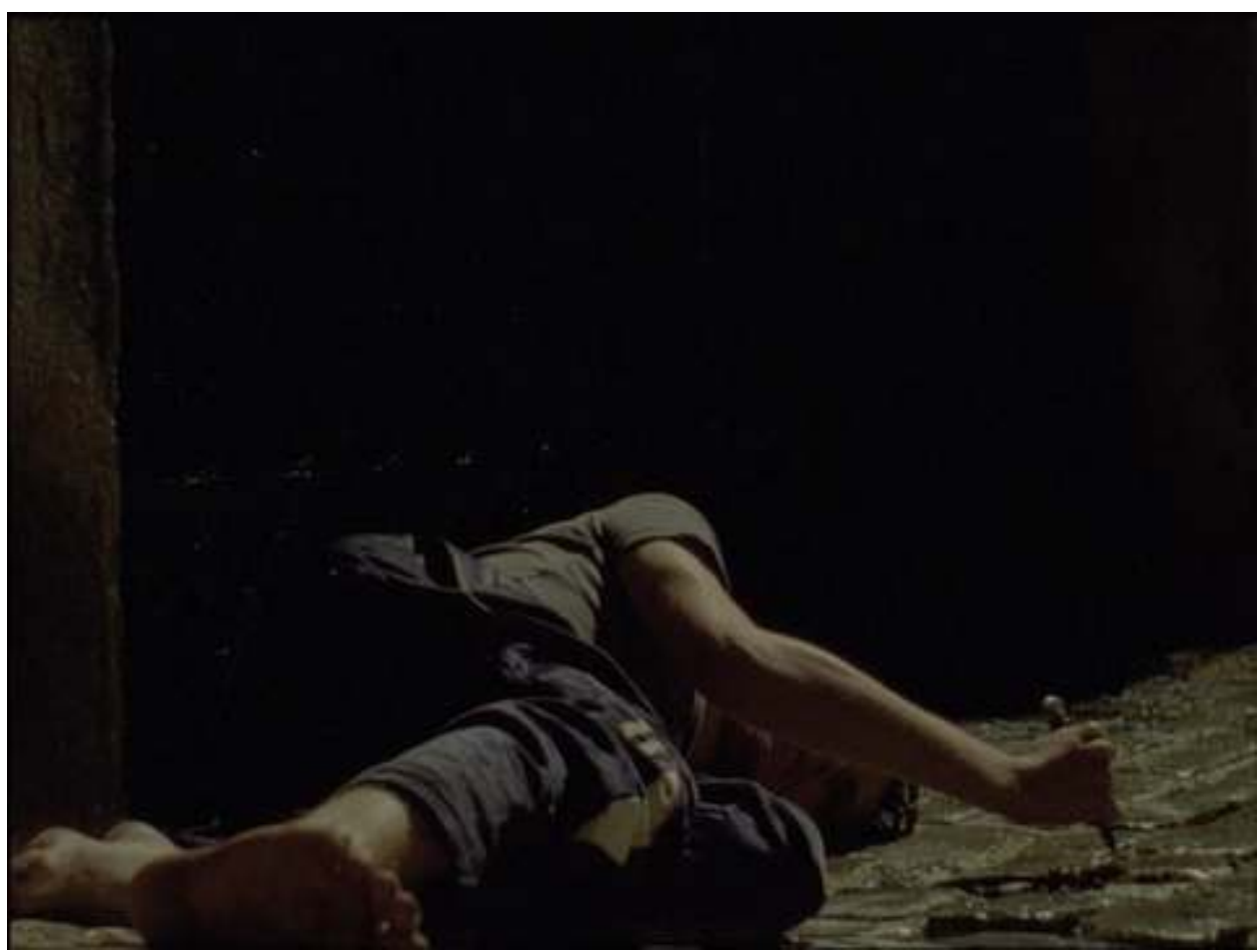

FIg. 2 : MOURIR COMME UN HOMME, JOAO PEDRO RODRIgUES (2009)

9 Le ténébrisme pictural du XVII ${ }^{e}$ siècle, celui du Caravage, de Rembrandt ou de Georges de La Tour, a souvent inspiré les cinéastes pour composer et éclairer des scènes nocturnes : la netteté de ses clairs-obscurs contraste fortement avec la pénombre homogène et 
conventionnelle de la nuit américaine, détache les figures, dramatise la scène. La reprise formelle a pu parfois verser dans le maniérisme, qui a peut-être atteint son extrême limite quand deux cinéastes habitués de la citation picturale se sont lancés dans la biographie d'un maître de cette époque : je pense aux ténèbres dans lesquelles sont plongés le Caravaggio de Derek Jarman (1986) et La Ronde de nuit de Peter Greenaway (2008). L'influence du Caravage sur le cinéaste Joao Pedro Rodrigues (O fantasma, Odete) est à la fois plus complexe et plus profonde que la simple reprise d'une manière, d'un style : Mourir comme un homme (Morrer como um homem, 2010, Portugal), film sur les marginalités sociales et sexuelles, repose la question caravagesque d'un relèvement du voir par le toucher afin de présentifier la chair au sein même d'un art visuel.

Une scène du film du jeune cinéaste lisboète évoque plastiquement Le Caravage : Rosario, l'amant toxicomane de Tonia, l'héroïne transsexuelle du film, fait une overdose ; sa chair blanche, éclairée froidement par la lune, s'étale depuis le bord du cadre jusqu'à une grande ombre murale; apparaissant en raccourci, allongé de trois quarts, son corps évoque L'amour endormi (1608) (fig.4); puis, quand Tonia vient le soulever (fig.3), la composition rappelle L'extase de Saint-François (1594-5) (fig.5). Mais c'est une autre scène qui prolonge plus profondément la pensée caravagesque de la tactilité du visible et de son lien avec la puissance pathétique des chairs ouvertes en mettant en scène un baiser sur la plaie.

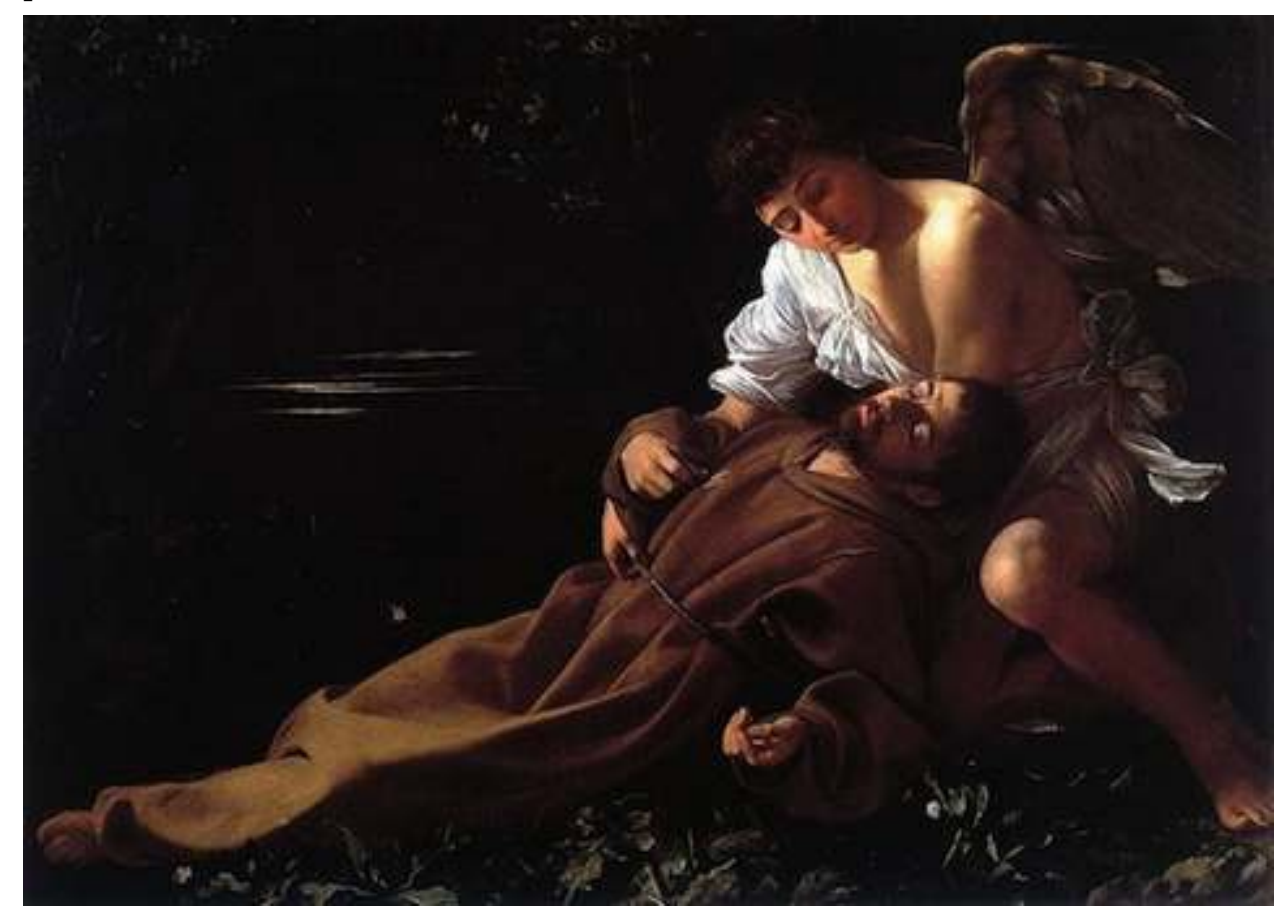

Fig. 5 : L'extase de Saint-François, Le Caravage (1594-5) 


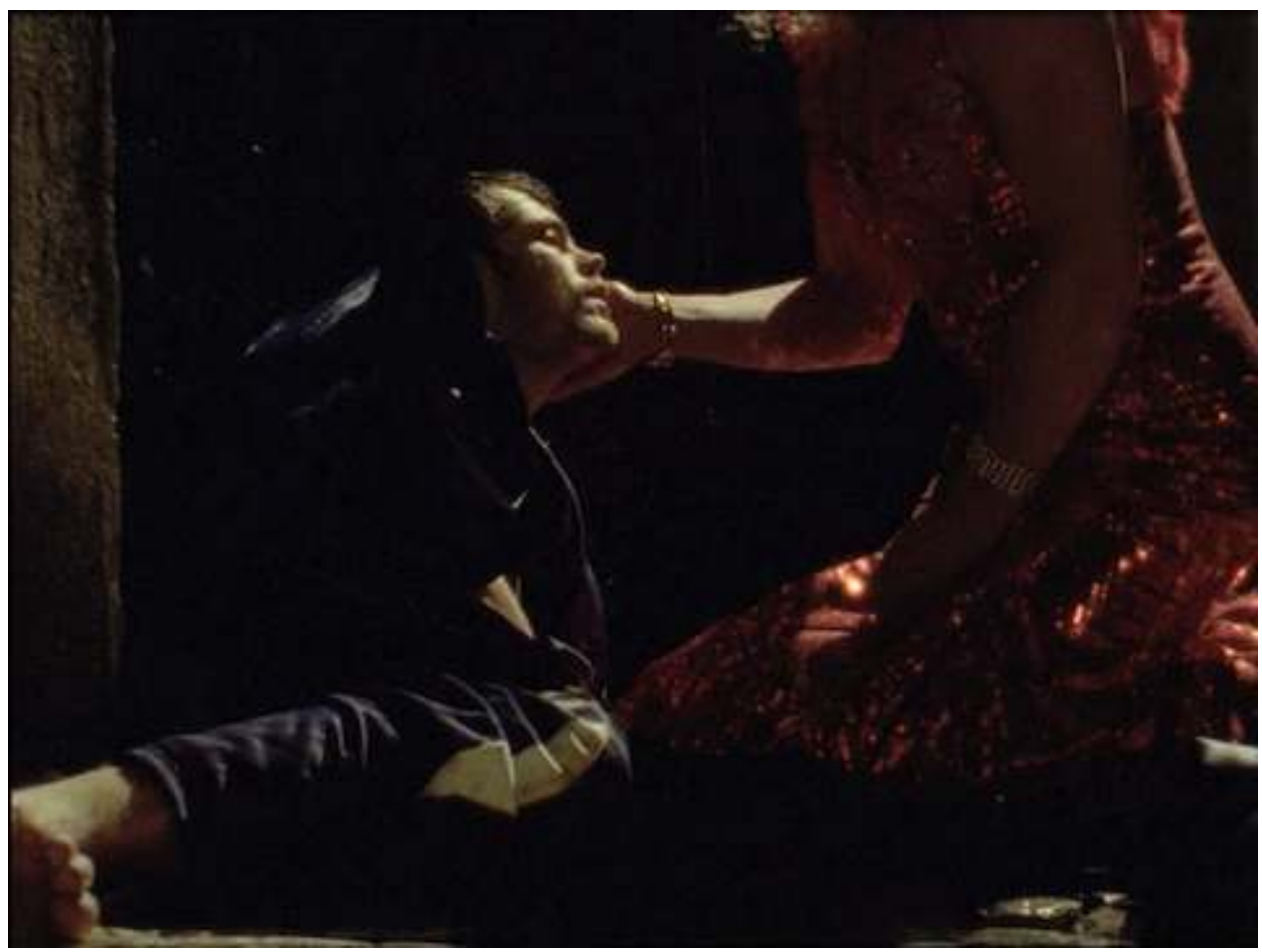

Fig. 6 : Mourir comme un homme, Joao Pedro Rodrigues (2009)

11 Le Caravage avait doublé sa représentation du toucher du stigmate costal par la figuration virtuelle d'un baiser de Thomas sur la main de Jésus, sur la marque du clou ; c'est peutêtre cela qui explique que Georges Didi-Huberman rapproche, sans citer le tableau, la scène de l'Évangile de Jean d'une scène de baiser ensanglanté dans un récit de Georges Bataille, Madame Edwarda ${ }^{6}$, mais aussi du baiser de Saint-François sur les plaies du lépreux et de celui que posa Catherine de Sienne sur le stigmate de la côte du Christ quand il lui apparut. Didi-Huberman trace ainsi la lignée littéraire, depuis un évangile jusqu'à une nouvelle érotique du $\mathrm{XX}^{\mathrm{e}}$ siècle, en passant par un récit hagiographique et un traité mystique, d'une formule de pathos ${ }^{7}$, c'est-à-dire le retour dans la longue durée d'un geste archaïque, violent et pathétique. C'est cette hypothèse d'une survivance de cette formule de pathos dans les arts visuels qui permet d'envisager le lien entre la toile du Caravage au film de Rodrigues comme le passage d'une virtualité du tableau à sa reprise paradoxale et explicite.

12 Tonia, déchirée entre le désir de se faire opérer pour devenir enfin physiquement la femme qui plairait à son amant et la peur de commettre un sacrilège en transformant le corps que Dieu lui a modelé, travaille dans une boîte de nuit où elle est la vedette d'un show de travestis; ce soir-là, malgré la disparition de son amant Rosario, elle part travailler. Dans le grand miroir devant lequel elle se maquille, apparait le reflet du corps sans visage, irréellement musclé, d'un performeur du cabaret, déguisé en maître sadomasochiste (Fig. 6). Après un insert montrant un gros plan du pied d'une collègue de Tonia en train d'enfiler un talon haut, le stripteaser, sommé de chercher Rosario, passe sa tête masquée entre les rideaux pour regarder dans la salle du cabaret, qui reste invisible au spectateur. C'est que cette loge, l'arrière-scène de la fabrique du spectacle, est déjà une scène, un univers spéculaire fait de fétiches et d'idoles, de reflets et de projections, le lieu d'une pure visibilité fantasmatique, d'une fantasmagorie de corps-images désincarnés. À l'avant-scène du tableau du Caravage répond donc l'arrière-scène d'une salle de spectacle 
invisible. Les immenses miroirs y redoublent le devenir spéculaire de corps maquillés, travestis, transformés en clichés, ou, plus encore, en purs simulacres : en images factices de ce qu'ils ne seront jamais (des reines, des vierges). Les fétiches dont Tonia s'entoure, que ce soient les posters érotiques ou les images sulpiciennes, confirment cette même artificialité de l'image: ce sont les représentations de corps idéalisés, modèles inatteignables de féminité ou parangons fantasmés de virilité ; ils participent d'une même idolâtrie, d'un même amour de l'image qui interdit tout en deçà (la chair) ou tout au-delà (le retrait, la distance, la face invisible du divin) au visible.

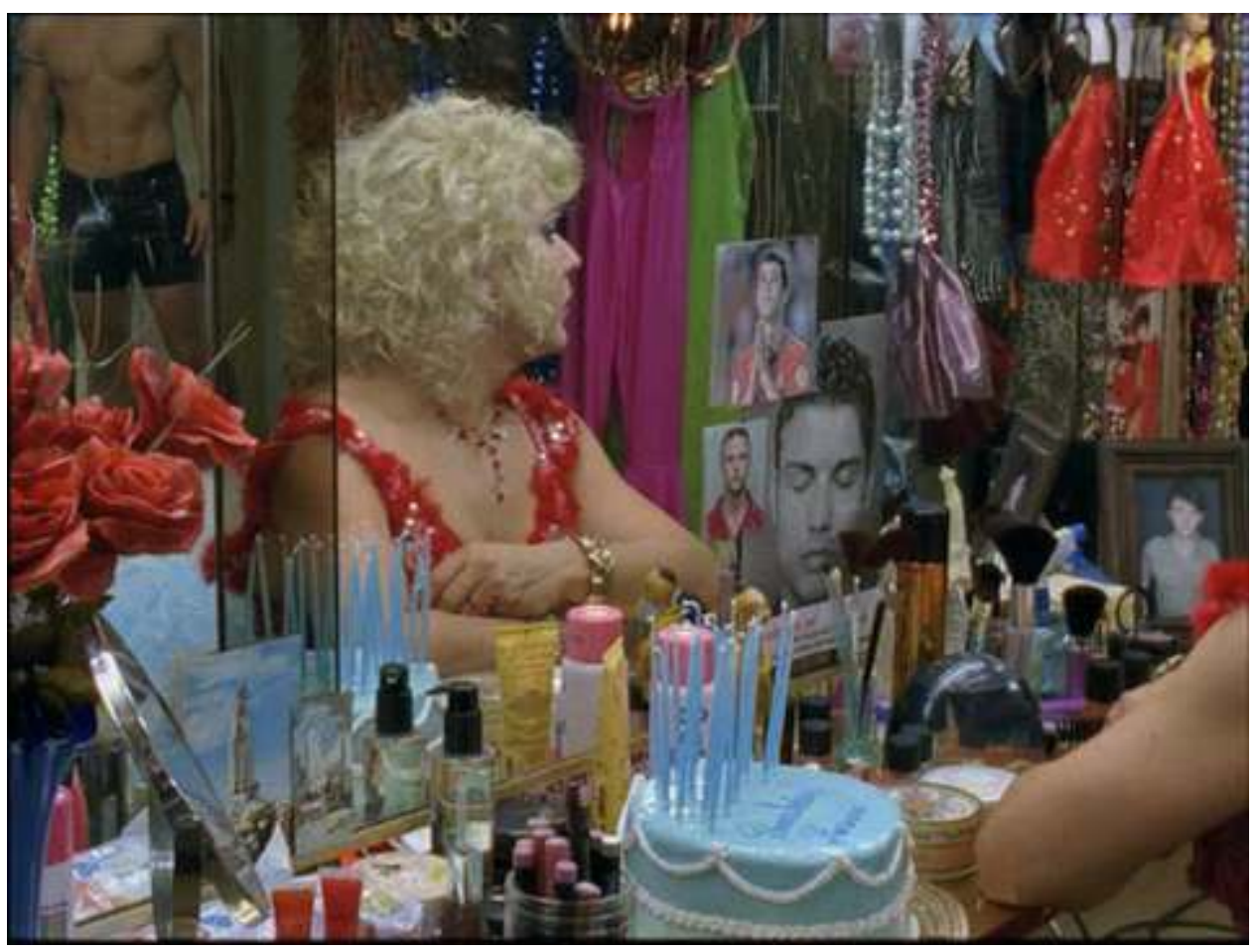

FIg. 6 : MOURIR COMME UN HOMME, JOAO PEDRO RODRIgUES (2009)

13 Jenny, la rivale de Tonia sur scène, plus jeune, plus belle et surtout opérée, la provoque en riant du départ de Rosario, avant de lui demander de l'aider à remonter la fermeture éclair de sa robe. Les deux femmes sont séparées en plan large par la paroi que forme un long miroir ; Tonia vient rejoindre de l'autre côté de ce miroir Jenny, qui l'attend de dos. Son large corps nous cache le dos de Jenny quand Tonia la blesse avec la fermeture éclair ; on entend juste un cri de douleur. Un faux raccord montre alors Tonia déjà accroupie, désormais à gauche du corps de Jenny, embrasser la plaie de Jenny et tourner son visage vers la caméra, la bouche pleine de sang. Le faux raccord, en plus de chambouler l'espace, a englouti quelques secondes, sautant un cran et blessant le regard, presque à la manière de ce zip qui a déraillé; désormais, le temps semble être suspendu : le visage de Tonia et le corps de Jenny restent immobiles, comme posant (fig.7). Ces deux plans font figure : il y a dans cette blessure plus à voir qu'un accident de travail ; le montage souligne, s'il était nécessaire (Tonia embrasse celle qu'elle vient de blesser et qu'elle déteste), que le geste du personnage n'a aucune logique fictionnelle ; son regard insistant en notre direction, sans être tout à fait un regard-caméra, indique qu'il s'y joue quelque chose d'important. 


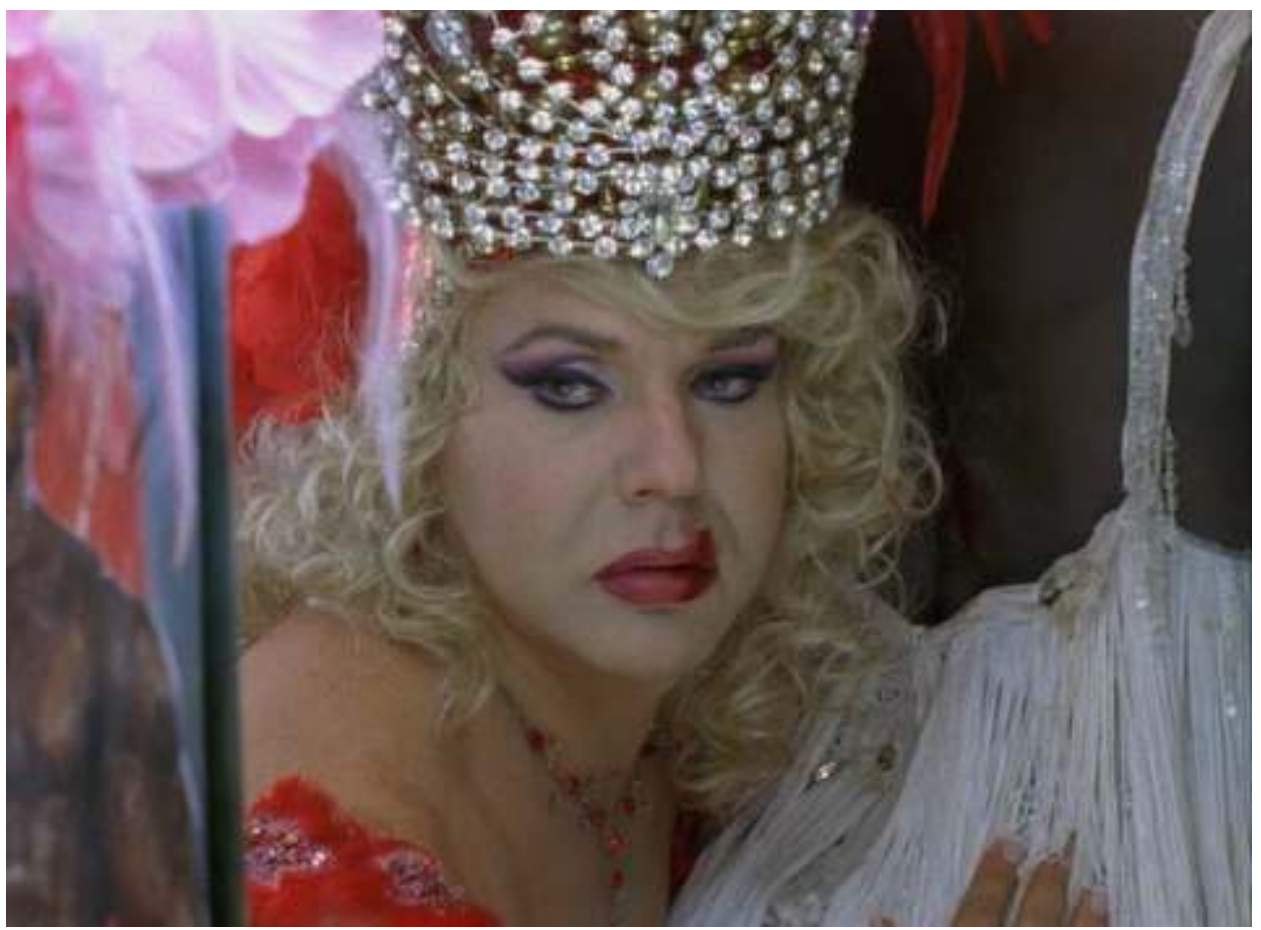

Fig. 7 : Mourir comme un homme, Joao Pedro Rodrigues (2009)

La violence physique et morale de la vaginoplastie que doit subir Tonia était dissimulée par la neutralisation scientifique du discours médical dans le générique du film, dans laquelle les mains d'un médecin modélisaient l'opération par un origami ; elle ressurgit ici, par détour, glissement et déplacement, selon une logique figurale. La blessure castratrice que craint Tonia, l'hérö̈ne l'inflige symboliquement à sa collègue. Or, Jenny est une transsexuelle ayant déjà subi une vaginoplastie. L'incident peut être lu comme une agression; Tonia, jalouse, écorche le corps qu'elle désire avoir. Mais aussi comme l'enquête d'un corps qui sonde un autre corps: la blessure est la métonymie de la castration; Tonia l'embrasse comme pour s'approcher au plus près de cette réalité, comme pour communier avec la chair souffrante, la chair ouverte qui est le lot de tous les transsexuels. La violence du faux-raccord, l'étrangeté du baiser, la solennité du regard de Tonia et la défaite de son visage ensanglanté, tout concourt ici à figurer l'irruption d'une visibilité nouvelle, jusqu'ici cachée, et ouvrir le plan à une dimension sacrée, mystique. À gauche du plan, un bout du miroir où est collé le poster d'un corps bodybuildé vient signifier que deux régimes de visibilité et de corporéité s'affrontent tout à coup. Le baiser sur la plaie est venu percer la surface d'un corps exhibé pour faire surgir une humeur extraite de la profondeur de la chair ; ce surgissement défait l'image parfaite d'une peau lisse pour donner à voir le corps réel de toute image : une profondeur sensible et informe que le spectacle dissimule.

15 Le film figure alors le dilemme le plus profond de Tonia : continuer à imiter, à jouer, à performer les femmes, avec pour modèle inaccessible la Vierge, et comme régime d'imagéité la représentation mimétique, sur le mode burlesque de la caricature ; ou franchir le pas, et s'incarner réellement dans un corps castré, ouvert, retourné. Ce dilemme psychologique est aussi un problème de théologie et de figurabilité, celui que Georges Didi-Huberman a posé à travers sa lecture de Tertullien, et qu'il résume ainsi :

" A travers le corps à corps de l'imitation et de l'incarnation, quelque chose passe, chez Tertullien, sans jamais être théorisé, sans jamais être rendu tout à fait clair ; 
quelque chose qui nous raconterait comment le visuel s'arrache du visible. Le visible, c'est le monde de l'idolâtrie, monde où l'image s'exhibe, se met en représentations, en spectacles ignobles, en concupiscences satisfaites. Le visuel, au contraire, c'est ce qui se voit au-delà, dans l'au-delà. Le visuel caractérise un monde où l'image est en présence et en promesse tout à la fois - bref, en aura, matière de l'âme ${ }^{8}$."

Didi-Huberman appelle le conflit du visible et du visuel recoupe l'opposit corps-image, du corps-spectacle et du corps incarné, de la chair ; ou, pour le dire dans les termes de Michel Henry, la dualité de tout corps humain, d'une part « corps chosique exposé dans le monde » et de l'autre " chair réelle », « chair vivante ${ }^{9}$ ». Ce conflit redouble la lutte théologique qui opposa Tertullien au docétisme défendant l'immatérialité spirituelle de Jésus. La question de figurabilité qui se pose, c'est celle de la possibilité d'incarner une image, d'imager de la chair, la chair comme matière animée, vivante, spirituelle. Or, Didi-Huberman explique que « le sang qui jaillit (...) dénote exactement ce qu'on pourrait nommer l'incursion du visuel dans le visible: soit, ici, l'irruption du dedans - l'irruption de la couleur viscérale, de la couleur d'âme, dans un monde visible d'où âme et viscère sont normalement retranchés, repliés dans les fonds de la chair ${ }^{10}$ ». Le sang qui jaillit de la blessure de Jenny et contamine la bouche de Tonia, c'est la matière commune d'une même souffrance charnelle, le partage de deux âmes qui souffrent de leur incarnation; et ce baiser est un rituel liturgique, le geste rejoué de la communion catholique. "Ceci est notre sang" dit Tonia de son regard inquiet tourné vers nous. Surtout qu'en embrassant la plaie de Jenny, elle a mêlé son rouge à lèvre au sang de son amie, et a donné à sa blessure la forme de deux lèvres entrouvertes, motif archétypal de la représentation christique des stigmates, autour duquel s'organisait déjà le tableau du Caravage, mais évoquant ici l'image de la vulve.

Du dogme de l'incarnation découle le drame de la vision: voir est toujours insuffisant, suspect, trompeur, sujet au simulacre, tant que la vision n'est pas compassion; puisque le Christ n'est pas une image, mais une chair réelle, souffrante, voir signifie croire en ce qui se dérobe à tout visible, c'est-à-dire en la réalité intérieure de sa chair ; la vision est toujours tendue vers le toucher, et, plus encore, vers l'acte de manger (Didi-Huberman parle d' "œil vorace »). Cet idéal d'incorporation a un versant pathétique et obscène, le baiser sur la plaie. Rejouer une telle scène semble permettre à Tonia de dépasser l'imitation fantasmatique de la Vierge, d'abandonner l'idolâtrie qu'elle porte à son image sulpicienne, pour vivre réellement la souffrance charnelle qui est, dans le catholicisme, la souffrance de toute âme incarnée; et plus particulièrement celle de Marie et de MarieMadeleine face au stigmate de l'autre aimé - d'un Dieu qui s'est fait chair saignante, ouverte, chair à vif, chair mourante.

Du tableau au film, de l'avant-scène obscure du tombeau à la coulisse spéculaire d'une scène demeurée hors-champ, la chair blessée demeure ce qui se dérobe au spectacle du monde visible et demande le geste obscène d'un baiser pour être figuré. Le seul spectacle que peut donner la chair, c'est la chorégraphie compassionnelle des corps s'agenouillant, se regroupant, se touchant et se baisant pour célébrer leur même essence charnelle dans le partage d'une communion. 


\section{NOTES}

1. J. Derrida, Le toucher, Jean-Luc Nancy, Paris, Galilée, 2000.

2. Tertullien, La chair du Christ, Paris, Les Éditions du Cerf, 1975. Voir aussi E. Falque, Dieu, la chair et l'autre. D'Irénée à Duns Scot, Paris, PUF, 2008, notamment le chap. «La consistance de la chair (Tertullien) », pp. 251-288.

3. C. Puglisi, Caravage, Paris, Phaidon, 2005, p. 216.

4. L. Marin, Détruire la peinture, Paris, Flammarion, 1997 ( $2^{\mathrm{e}}$ édition), p. 201.

5. Ibid., pp. 204-205.

6. «Enfin, je m'agenouillai, je titubai, et je posai mes lèvres sur la plaie vive ", raconte le narrateur à propos d'une prostituée qui lui affirme dans le même temps : «Tu vois (...) je suis Dieu ». G. Bataille, Madame Edwarda, œuvres complètes, Paris, Gallimard, 1970-1988, tome III, p. 20, cité par G. Didi-Huberman, L'image ouverte, Paris, Gallimard, 2007, pp. 336-337.

7. A. Warburg, Essais Florentins, Paris, Klincksieck, 1990. Voir aussi l'analyse du concept par G. Didi-Huberman, L'image survivante. Histoire de l'Art et Temps des Fantômes selon Aby Warburg, Paris, Les Éditions de Minuit, 2002, notamment pp. 191-223, et par G. Agamben, Image et Mémoire. Écrits sur l'image, la danse et le cinéma, Paris, Desclée de Brouwer, 2004, pp. 9-69

8. G. Didi-Huberman, L'image ouverte. Motifs de l'incarnation dans les arts visuels, op.cit., p.119.

9. M. Henry, Incarnation. Une philosophie de la chair, Paris, Les Éditions du Seuil, 2000, p. 310.

10. G. Didi-Huberman, L'image ouverte, op. cit., p. 134.

\section{RÉSUMÉS}

L'épisode de l'incrédulité de Saint Thomas dans l'Évangile de Jean est l'indice d'un recouvrement du toucher par le voir dans la pensée chrétienne. La représentation de l'anecdote par Le Caravage figure cet échec de l'œil relayé par la main, et redouble le toucher de la plaie costale par un baiser sur la marque du clou. Nous étudions la reprise de ce geste, compris comme «formule de pathos ", dans un film contemporain de Joao Pedro Rodrigues, où une transsexuelle angoissée par sa vaginoplastie programmée embrasse la plaie d'une rivale déjà opérée. Ce baiser ensanglanté est compris comme la communion obscène de deux chairs ouvertes unies par une même souffrance.

\section{AUTEUR}

\section{OLIVIER CHEVAL}

Normalien, certifié de Lettres Modernes, élève de l'École Nationale des Beaux-Arts de Paris, Olivier Cheval est depuis septembre 2012 doctorant contractuel sous la direction de Luc Vancheri au laboratoire Passages XX-XXI de l'université Lyon 2. Il est l'auteur d'une note de lecture sur L’Hypnose au cinéma de Raymond Bellour dans le n³61 de la revue Esprit. 